JMB

\title{
Structural Investigations of Calcium Binding and its Role in Activity and Activation of Outer Membrane Phospholipase A from Escherichia coli
}

\author{
H. J. Snijder ${ }^{1}$, R. L. Kingma ${ }^{2}$, K. H. Kalk', N. Dekker², M. R. Egmond ${ }^{2}$ \\ and B. W. Dijkstra1*
}

${ }^{1}$ Laboratory of Biophysical Chemistry, BIOSON Research Institute, University of Groningen, Nijenborgh 4 9747 AG, Groningen, The Netherlands

${ }^{2}$ Department of Enzymology and Protein Engineering Centre for Biomembranes and Lipid Enzymology, Institute of Biomembranes, Utrecht University, Padualaan 8 $3584 \mathrm{CH}$ Utrecht, The Netherlands

\begin{abstract}
Outer membrane phospholipase A (OMPLA) is an integral membrane enzyme that catalyses the hydrolysis of phospholipids. Enzymatic activity is regulated by reversible dimerisation and calcium-binding. We have investigated the role of calcium by X-ray crystallography. In monomeric OMPLA, one calcium ion binds between two external loops (L3L4 site) at $10 \AA$ from the active site. After dimerisation, a new calcium-binding site (catalytic site) is formed at the dimer interface in the active site of each molecule at $6 \AA$ from the L3L4 calcium site. The close spacing and the difference in calcium affinity of both sites suggests that the L3L4 site may function as a storage site for a calcium ion, which relocates to the catalytic site upon dimerisation. A sequence alignment demonstrates conservation of the catalytic calcium site but evolutionary variation of the L3L4 site. The residues in the dimer interface are conserved as well, suggesting that all outer membrane phospholipases require dimerisation and calcium in the catalytic site for activity. For this family of phospholipases, we have characterised a consensus sequence motif (YTQ- $\mathrm{X}_{n}-\mathrm{G}-\mathrm{X}_{2}$ $\mathrm{H}-\mathrm{X}-\mathrm{SNG}$ ) that contains conserved residues involved in dimerisation and catalysis.
\end{abstract}

(C) 2001 Academic Press

Keywords: phospholipase; membrane enzyme; calcium-binding; dimerisation; crystal structure phospholipases or lipases, ${ }^{3,6-8}$ but appears to be the prototype of a unique family of outer membrane enzymes occurring in a large variety of Gram-negative bacteria.

The physiological role of OMPLA is not conclusively known, ${ }^{9}$ although in various bacteria functions have been established for this enzyme. In E. coli OMPLA is involved in the excretion of bacteriocins. ${ }^{10-13}$ In Campylobacter coli, OMPLA is a major haemolytic factor. ${ }^{8}$ Recently, Dorrell et al. ${ }^{14}$ demonstrated involvement of OMPLA in the invasion of the gastric mucosa by Helicobacter pylori and postulated a role in causing tissue damage after colonisation.

Although OMPLA is constitutively expressed in E. coli, no activity is detected under normal conditions. ${ }^{15,16}$ Activity is observed only after events that disrupt membrane integrity, such as temperature shock, phage-induced lysis, or colicin release. ${ }^{10-13,17,18} \mathrm{~A}$ strong induction of OMPLA activity and concomitant extensive breakdown of the outer membrane has lethal consequences for 
the cell. $^{18}$ This detrimental effect of OMPLA necessitates a strict regulation of its activity. Chemical cross-linking studies demonstrated that activity is regulated by reversible dimerisation in a calcium and substrate-dependent fashion. ${ }^{19,20}$ Calcium or substrate alone is sufficient for dimerisation. ${ }^{19}$ Two calcium-binding sites with different binding affinities were characterised per monomer, with each site having supposedly distinct functions in dimerisation and in catalysis. ${ }^{21}$

Recently, we solved X-ray crystal structures of a calcium-free OMPLA monomer, and a calciumcontaining OMPLA dimer. ${ }^{22}$ The structure of OMPLA has a 12-stranded antiparallel $\beta$-barrel fold with a serine hydrolase-like catalytic triad consisting of Ser144, His142 and Asn156. In the dimer, the calcium ion (catalytic calcium ion) is observed at the dimer interface in the active site. It is located at a position suited to polarise the carbonyl group of the scissile ester bond of the substrate and to stabilise the negatively charged tetrahedral intermediate, thus explaining its essential role in catalysis.

Here, we present crystal structures of monomeric OMPLA with bound calcium, and calcium-free dimeric OMPLA. Surprisingly, the monomer OMPLA calcium-binding site is different from that in the dimer. These results enable us to postulate a detailed model for calcium-binding and activation.

\section{Results}

\section{Monomer and calcium-binding}

In the previously determined monomeric OMPLA structure, no calcium ion was identified. ${ }^{22}$ Soaking of the OMPLA monomer crystals for 30 minutes in a mother liquor with $50 \mathrm{mM} \mathrm{Ca}^{2+}$ added, resulted in the binding of calcium. The binding of calcium did not induce significant conformational changes. The r.m.s. coordinate difference for all 2094 common atoms of calcium-bound and calcium-free OMPLA is $0.63 \AA$. The r.m.s. coordinate difference for the 257 common $C^{\alpha}$ atoms is $0.30 \AA$, which is comparable to the coordinate error of both structures as estimated by sigmaA. ${ }^{23}$ The largest differences occur in the termini and loop regions, in particular residues 13, 57 and 181, and correlate with high crystallographic $B$-factors (Figures 1 and 2 ).

The calcium-binding site is different from the calcium-binding site observed in the dimeric OMPLA structure ${ }^{22}$ (see below). In monomeric OMPLA, the calcium ion is bound between loops L3 and L4 (L3L4 site) at a distance of approximately $10 \AA$ from the active-site serine residue. The carboxylate group of Asp184 ligates the calcium ion at a distance of $2.5 \AA$. Furthermore, the Asp149 carboxylate group is in the proximity of the calcium ion (Figure 3(a)). These two residues as well as the calcium ion have high crystallographic temperature factors (the average $B$-factors per residue are 71, 91 and $91 \AA^{2}$, respectively). However, the $B$-factors are in general agreement with the high $B$-factors observed in other loops in the structure. Data collected from crystals grown in the presence of $\mathrm{SmCl}_{3}$ instead of $\mathrm{CaCl}_{2}$, unequivocally confirm the cation-binding site. No other calciumbinding site besides the L3L4 binding site could be found in difference electron density maps of calcium-bound and calcium-free monomeric OMPLA or in the $\mathrm{SmCl}_{3}$ electron density maps.

Preliminary structural characterisation of an Asn156Ala mutant of OMPLA, which shows better diffraction, supports the binding mode of cal-

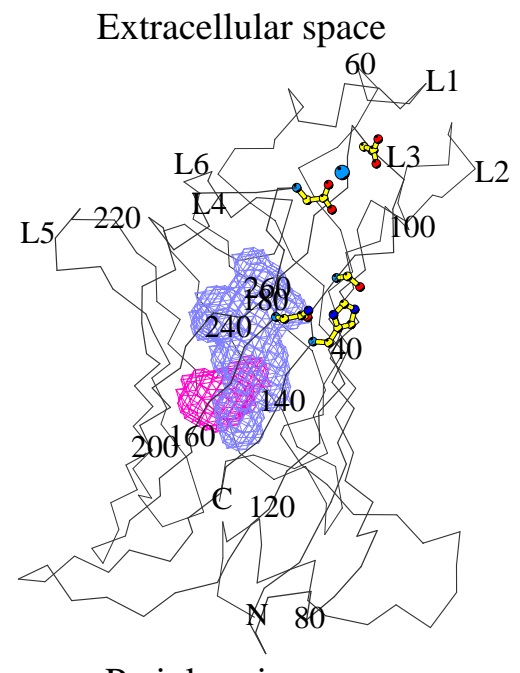

Periplasmic space

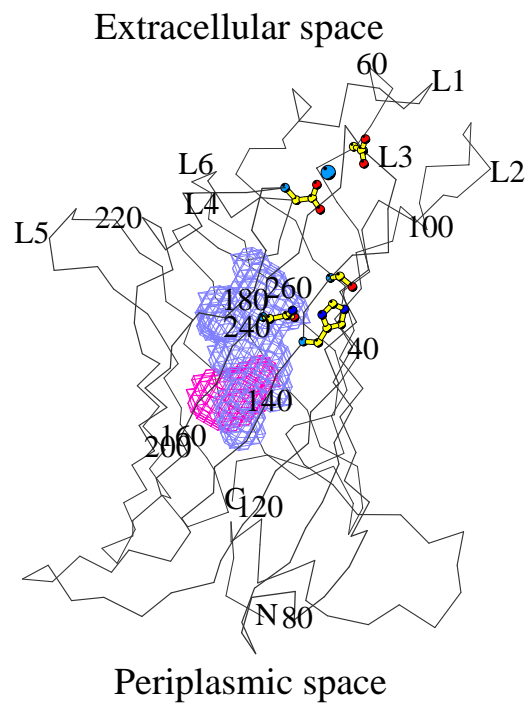

Figure 1. Stereo diagram of the structure of monomeric OMPLA shown as a $C^{\alpha}$ trace. Every 20th residue is labelled. OMPLA contains two internal cavities, which are indicated in blue and pink. The active-site residues and the two aspartate residues that ligate the calcium ion are displayed as ball-and-stick models, and the calcium ion is depicted as a blue sphere. The external loops are numbered consecutively from the $\mathrm{N}$ to the C terminus as L1 to L6. 


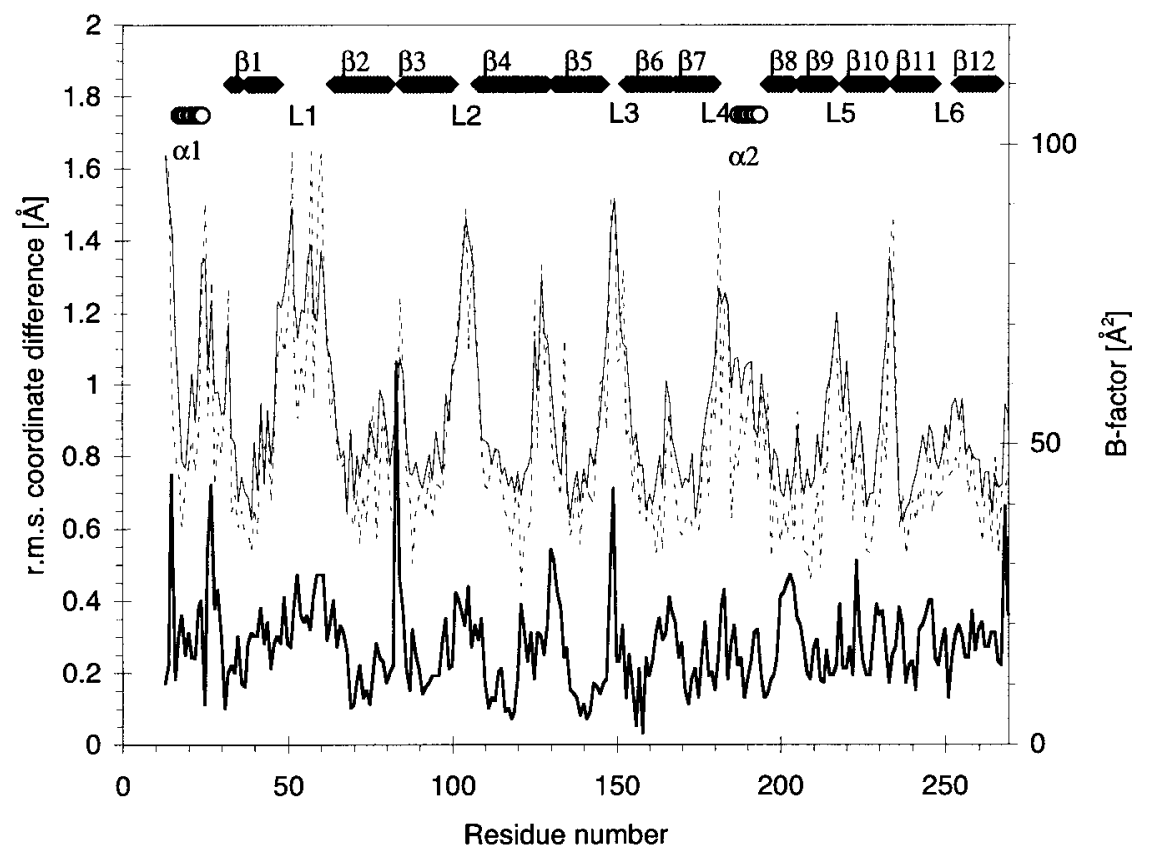

Figure 2. The r.m.s. coordinate difference of the common $C^{\alpha}$ atoms between calcium-free $e^{22}$ and calcium bound OMPLA (continuous dark line). $B$ values are depicted in the thin continuous and broken lines for calcium-bound and calcium-free monomeric OMPLA, respectively. Secondary structure elements are shown for residues in $\alpha$-helices $(\bigcirc)$ and $\beta$-strands $(\bullet)$.

cium in the L3L4 site (Figure 3(b)). The calcium ion is bound by one carboxylate oxygen atom of Asp149 and Asp184, at a distance of $2.46 \AA$. The calcium ion is complexed by the syn orbitals of the oxygen atoms. The angles between the calcium ion and the carbon-oxygen bond are $118.5^{\circ}$ and $125^{\circ}$ for Asp149 and Asp184, respectively. Thus, the two ligands display near-optimal ligation geometry. Additional ligands for the calcium ion may be provided by water molecules; however, these cannot be observed in the crystal structure owing to the modest resolution, high flexibility and disorder.

\section{Calcium-free dimer}

OMPLA activity is regulated by reversible dimerisation. ${ }^{19,20}$ OMPLA monomers associate in a parallel fashion via the flat barrel surfaces to form dimers (Figure 4). This dimerisation results in the formation of two hydrophobic, substrate-binding clefts, which are created along the subunit interface parallel with the $\beta$-barrel axis. In the dimeric structure, we had previously identified one calciumbinding site per monomer (catalytic calcium) located at the interface of the dimer. The calcium ion is bound in the active site by ligands from both monomers, i.e. the $\mathrm{O}^{\gamma}$ atom of Ser152, the mainchain carbonyl oxygen atom of residue 147 and the main-chain carbonyl oxygen atom of residue 106 from the other monomer. ${ }^{22}$ Crystals of the dimer grown in the absence of calcium show that the structure of the calcium-free form of the dimer is virtually identical with the calcium-complexed dimer, and that no calcium ion is present at this site. The r.m.s. coordinate difference between the two structures is only $0.23 \AA$ for the 448 common $\mathrm{C}^{\alpha}$ atoms. The coordinate differences for all atoms of the calcium ligands in the dimer are within the experimental errors of the structures. Thus, the catalytic calcium-binding site is preformed in the OMPLA dimer and calcium-binding at this site does not require conformational changes (Figure 3(c) and (d)).

\section{Differences between the monomeric and dimeric OMPLA}

The previously determined calcium-free monomer and calcium-bound dimer displayed small but significant differences in the orientation of the active-site residues His142 and Asn156. Whether these differences arose from calcium-binding or inhibition and dimerisation could not be determined. With the structures described here, it can now be established that the arrangement of the active-site residues is independent of calcium-binding in both monomeric and dimeric OMPLA. Instead, the active-site histidine residue in the dimer structure has rotated towards Asn156 because of a close contact ( $2.7 \AA$ ) with the sulfonyl $\mathrm{O} 2$ of the inhibitor. This interaction can be thought to mimic the interaction of the imidazole group with the natural substrate. Such an interaction is favourable for the transfer of a proton from the imidazole group to the leaving group of the sub- 


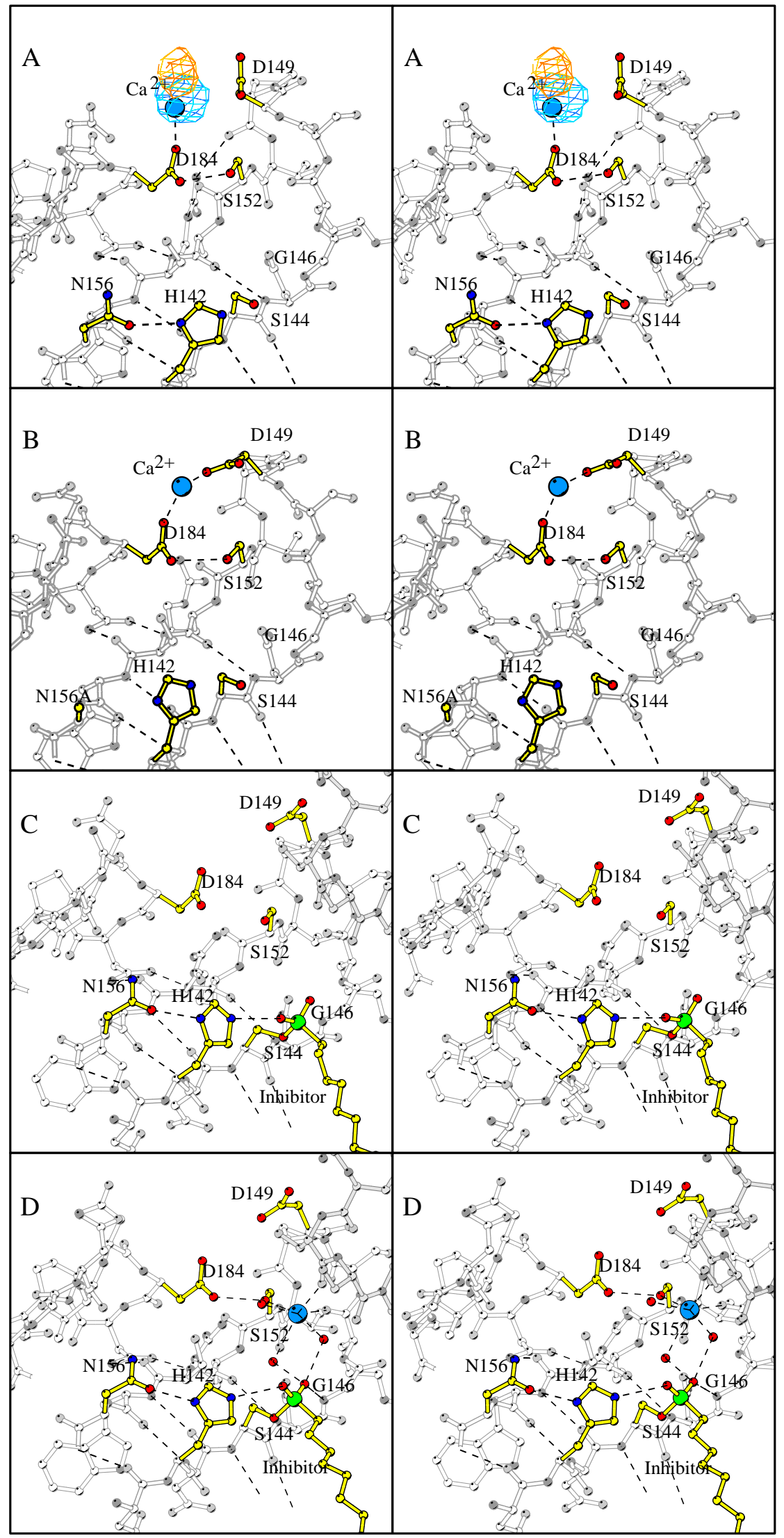

Figure 3. Stereo view of the $\mathrm{Ca}^{2+}$-sites of OMPLA in ball-andstick representation. The active-site residues, the inhibitor and residues involved in calcium-binding are highlighted. Calcium is shown as a large blue sphere. Hydrogen-bonding interactions are marked with broken lines. (a) Calcium binding in monomeric OMPLA. The orange electron density map shows the anomalous electron density signal of samarium (contoured at $4 \sigma$ ). The blue electron density presents the isomorphous difference electron density of the calcium-bound and unliganded monomeric OMPLA. Both maps were calculated using phases obtained from the refined unliganded monomeric OMPLA model. ${ }^{22}$ (b) Calcium binding in the monomeric form of the Asn156Ala OMPLA mutant. (c) The empty calcium-binding site in dimeric OMPLA. (d) Calcium binding in the dimeric form of OMPLA. 


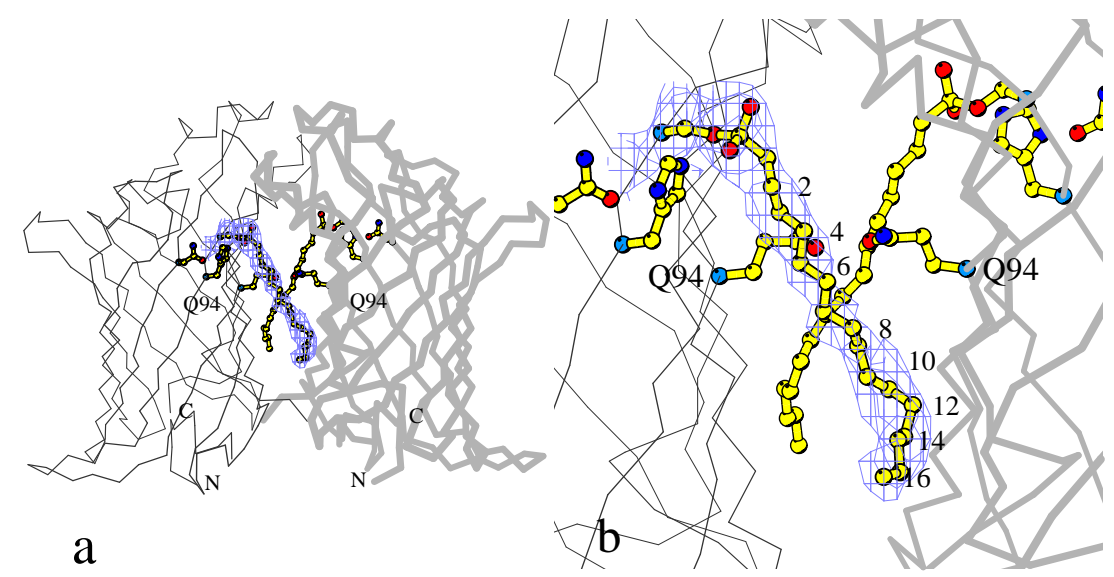

Figure 4. Wire model of the OMPLA dimer. (a) Overview of the OMPLA dimer. (b) Detail of the active site with the inhibitor. The active-site residues (His142, Ser144 and Asn156) and the key dimerisation residue (Gln94) are depicted in ball-and-stick representation. Electron density is shown around one of the inhibitors (sigmaA-weighted omit density contoured at the $1 \sigma$ level). The enzyme has reacted with the inhibitor hexadecanesulfonylfluoride, which is clearly indicated by continuous electron density for the nucleophilic Ser144 and the inhibitor's sulfonyl sulphur atom. Every second carbon atom of the inhibitor is labelled.

strate, thereby facilitating departure of the leaving group and thus stimulating substrate hydrolysis. Comparable interactions have been described in lipases complexed with substrate analogues, ${ }^{24-26}$ which were proposed to similarly facilitate departure of the leaving group of the enantio-preferred substrate.

\section{Sequence comparison}

The pldA gene coding for OMPLA is widespread among Gram-negative bacteria. An alignment of 16 complete OMPLA sequences is depicted in Figure 5. In these sequences, only 20 amino acid residues are absolutely conserved and another 17 are similar, thus yielding a homology of only $13.5 \%$. The signal sequences and $\mathrm{N}$-terminal region show the largest variations and prove difficult to align. Further variations and insertions are concentrated in the loop and turn regions, with the most variable part located in loop L1, which covers a substantial part of the extracellular exposed surface. Residues located in $\beta$-strands that face the exterior show marginally more variation (average variation $43 \%, 81$ residues) than those located inside the $\beta$-barrel (average variation 39\%, 65 residues). This pattern of conservation has been observed for other outer membrane proteins amongst others $\mathrm{OmpA}^{27}$ and the general porins. ${ }^{28}$

The best conserved residues are the active-site residues His142 and Ser144, and the loop participating in the oxyanion-hole formation (Asn145 and Gly146). The evolutionary variation of the third catalytic residue, Asn156, is remarkable. Sitedirected mutagenesis demonstrated a modest role of Asn156 in catalysis, which could account for the observed evolutionary variation. ${ }^{29}$
The L3L4 calcium site is not absolutely conserved. Residue Asp149 is an arginine residue in Neisseria species, and a glycine residue in the Helicobacter strains. The second calcium ligand, Asp184, is conserved in all but the Helicobacter strains, where it is serine. In contrast, the catalytic calcium site seems conserved among all sequences. Ser152, which ligates the calcium ion with its hydroxyl group, is absolutely conserved. The other protein ligands are the main-chain carbonyl oxygen atoms of residues Ser106 and Arg147. Conservation of residues in the vicinity of the catalytic calcium-binding site suggests a similar local structure and thus conservation of the position of the carbonyl groups of residues 106 and 147 .

The catalytic calcium-binding site has ligands from both monomers in the dimer, thus calcium may stabilise the dimeric complex. Other residues that stabilise the dimer are conserved. For example, proline 108 locally optimises the peptide mainchain conformation for making intermolecular hydrogen bonds in the dimer. The other invariant residues, Tyr92 and Gln94, are part of a membrane-embedded polar patch that supplies specific intermolecular polar interactions in the dimer. Although other residues that contribute to this polar patch are variable, the patch retains it hydrophilic character in all pldA sequences.

Apart from the residues conserved in the active site and dimer interface, a number of residues are conserved for structural reasons. Three absolutely conserved residues are observed around the active site, Pro116, Gly139 and Asn185. Proline 116 sterically restricts the rotational freedom of the catalytic histidine residue. The rigid side-chain of the proline residue excludes $\chi_{1}$ angles of the histidine residue from approximately $-10^{\circ}$ to $+140^{\circ}$. The $\chi_{1}$ rotation of the imidazole group is further restricted by steric hindrance from the bulky side-chain of 

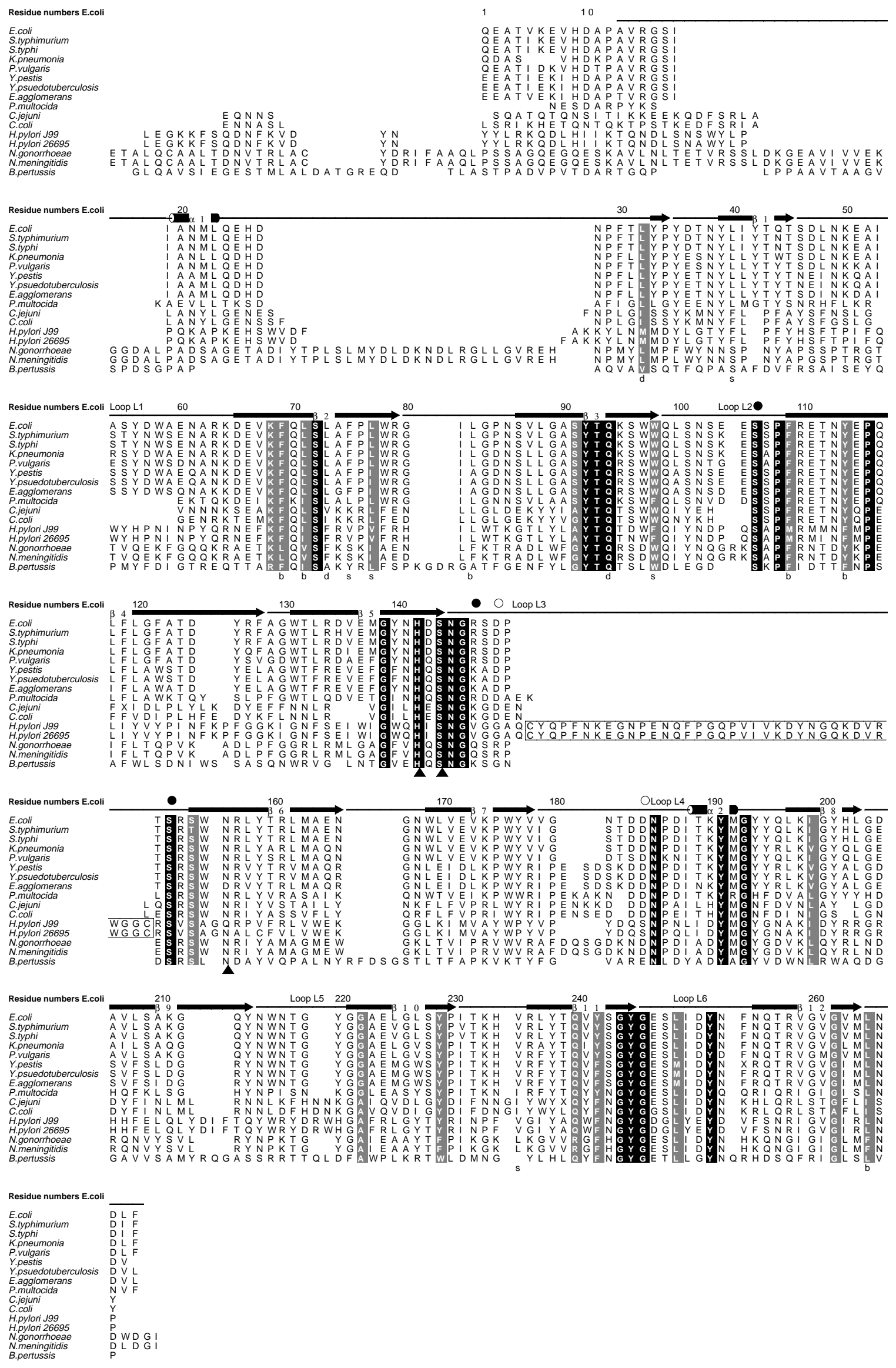

Figure 5 (legend opposite) 
residue 140, tyrosine in E. coli OMPLA. Residue Gly139 is located in the sharp bend of the $\beta$-strand, which also contains the catalytic His142 and Ser144. This glycine has phi-psi angles that fall outside the core regions for non-glycine residues, according to the definition given by Kleywegt \& Jones. ${ }^{30}$ The larger conformational freedom of glycine residues allows for the sharp bend of the $\beta$-strand, resulting in an ellipsoid cross-section of the $\beta$-barrel. The carboxamide moiety of residue Asn185 forms hydrogen bonds with the mainchain atoms of residue 154, effectively extending the $\beta$-barrel hydrogen bonding pattern of $\beta$-strand 6 , which is part of the active site. These residues are of structural importance to the active site, hence their evolutionary conservation.

Finally, an extensive conserved salt-bridge and hydrogen bonding network is present in the interior of the $\beta$-barrel between the highly invariant residues Glu66, Gln99, Arg110, Glu247 and Arg258. These residues are located in the region where the $\beta$-strands of the dimer interface pass into the loops. This extensive network of interactions fixes the $\beta$-strands and confers rigidity to the $\beta$-barrel. The importance of this network is illustrated by two point mutations, Glu66Pro and Glu247Gly, which are completely inactive. ${ }^{31} \mathrm{~A}$ second conserved internal interaction network is formed by Gln70, Ser72, Thr93 and Lys95, which supplies further structural stabilisation of the dimerisation face of the protein.

\section{Discussion}

The calcium requirement of OMPLA for activity has long been established. ${ }^{5}$ Biochemical studies have characterised two calcium-binding sites, one with a high calcium affinity and the other with tenfold lower affinity $\left(K_{\mathrm{d}} \approx 36 \mu \mathrm{M}\right.$ and $\left.358 \mu \mathrm{M}\right){ }^{21}$ Our results are in agreement with those studies and show that one $\mathrm{Ca}^{2+}$ site occurs in monomeric OMPLA (L3L4 site), and that the other is present exclusively in the active site of the dimeric enzyme (catalytic calcium site).

The calcium affinities of OMPLA in solution in the presence of zwitter-ionic phospholipid ana- logues are 1000-fold higher than the $\mathrm{Ca}^{2+}$ concentrations required for binding calcium in the crystal structures (15 and $50 \mathrm{mM} \mathrm{Ca}{ }^{2+}$ for dimeric and monomeric OMPLA, respectively). This higher affinity may originate from a strong dependency of the calcium affinity of the enzyme on the detergent or lipid environment. ${ }^{21}$ In the presence of zwitterionic phospholipid-like detergents, OMPLA has a calcium affinity in the micromolar range, three orders of magnitude higher than for non-ionic alkylpolyoxyethylene detergents. ${ }^{21}$ For the crystallisations a non-ionic detergent was used (1-O-noctyl- $\beta$-D-glucopyranoside, $\beta$-OG), which similarly may result in millimolar calcium affinity for OMPLA. The molecular basis of the low affinity is not known, but the different detergents might induce subtle conformational changes in the highly flexible loops of the enzyme. Alternatively, the detergents could alter the local electrostatic potential at the calcium-binding site and thereby modulate the calcium affinity.

In the crystal structure of dimeric OMPLA the catalytic $\mathrm{Ca}^{2+}$ site is preferentially occupied over the L3L4 site. Ubarretxena-Belandia et al. ${ }^{21}$ showed that the high-affinity binding site plays a role in dimer stabilisation, as dimerisation required only a low calcium concentration. As the catalytic calcium ion stabilises the dimer owing to its coordination by ligands from both monomers, we conclude that the catalytic site corresponds with the high-affinity calcium-binding in solution.

The catalytic calcium in the active site is in a position such that it can polarise the carbonyl bond of the ester group of the substrate and thus facilitate nucleophilic attack by Ser144. Moreover, the catalytic calcium ion can stabilise negatively charged reaction intermediates via two water molecules. ${ }^{22}$ In contrast, the precise role of the L3L4 calcium ion is unknown, Various possible functions can be considered. Modelling of a substrate in the active site of OMPLA shows that the phosphate moiety could, in principle, interact with the calcium ion at the L3L4 site. However, Horrevoets et $a l .{ }^{5}$ have already demonstrated that the presence of a phosphate group is not a strict substrate requirement. Moreover, Ubarretxena-Belan-

Figure 5. Sequence alignment of E. coli OMPLA and 15 homologous enzymes in descending order of similarity. Residues are numbered according to the mature E. coli OMPLA. Complete pldA sequences have been identified in Bordetella pertussis, Campylobacter coli, ${ }^{8}$ Campylobacter jejuni, ${ }^{50}$ Enterobacter agglomerans, ${ }^{7}$ E. coli, ${ }^{3}$ Helicobacter pylori $26695^{51}$ and Helicobacter pylori J99, ${ }^{52}$ Salmonella typhimurium, ${ }^{6}$ Klebsiella pneumonia, ${ }^{6}$ Neisseria gonorrhoeae, ${ }^{7}$ Neisseria menigitidis, ${ }^{53}$ Pasteurella multocida, Proteus vulgaris, ${ }^{6}$ Salmonella typhi, Salmonella enteriditis, Yersinia pestis, ${ }^{7}$ and Yersinia pseudotuberculosis. Fragments with substantial homology to the E. coli pldA sequence have been identified in Bordetella bronchiseptica, Shewanella putrefaciens and Salmonella paratyphi. Residues involved in calcium-binding are indicated above the alignment by open circles (L3L4 site) and filled circles (catalytic site). The active-site residues are indicated below the sequences by the filled arrows. Strictly conserved residues are shown in the black boxes, homologous residues in grey boxes. Residues involved in substrate binding and dimerisation are indicated by the lower-case letters below the alignment: substrate binding (s), dimerisation (d), and both substrate binding and dimerisation (b). A large insert in loop L3 is observed in the two H. pylori strains; it is indicated by a horizontal grey box. The OMPLA sequences of S. typhi and S. enteriditis are identical, only S. typhi is included in the Figure. 
dia et al. ${ }^{21}$ described that calcium dissociation constants are similar for substrates with and without phosphate groups. Taken together, it is unlikely that the L3L4 calcium ion is crucial for substrate binding.

Alternatively, the calcium ion could complex with phosphate groups from the lipopolysaccharide (LPS) matrix of the outer membrane. Recently, a complete LPS molecule was co-crystallised with the ferrichrome receptor FhuA and an LPS-binding motif was deduced.32 The LPS molecule runs parallel with the $\beta$-barrel axis of FhuA. Superimposing FhuA on OMPLA using the aromatic girdles of both proteins as a guide shows that the L3L4 calcium ion can interact with the phosphate groups of the lipid A moiety of LPS molecules. Such interactions may have a physiological function in tethering the protein to the LPS matrix, thus maintaining an impenetrable barrier in much the same way as LPS molecules interact via divalent cations. Thus, OMPLA that lacks the LPS-binding motif found in FhuA may have acquired an alternative way to complex LPS via the L3L4 calcium-binding site.

Finally, not conflicting with the above-proposed role, the L3L4 site could be a sink for calcium ions. The L3 $\mathrm{L}_{4} \mathrm{Ca}^{2+}$ site is more accessible to the solvent than the catalytic site, which is more deeply embedded in the protein. While in monomeric OMPLA only the low-affinity L3L4 site exists, in dimeric OMPLA two new high-affinity $\mathrm{Ca}^{2+}$-binding sites are present (one per monomer) at the dimer interface close to the active site. In view of the short distance between the L3L4 site and the catalytic $\mathrm{Ca}^{2+}$ site, a transfer of calcium from the L3L4 site to the high-affinity catalytic site upon dimerisation can be envisaged. In that way, a fully functional active dimer could be formed.

We propose a model for calcium-binding and activation of OMPLA (Figure 6) adapted from that proposed by Ubarretxena-Belandia. ${ }^{21}$ Normally, the enzyme resides in the outer membrane in the inactive monomeric form. The calcium concentration in the bacterial outer membrane environment is about 0.1 to $1 \mathrm{mM}^{33}$ and calcium is bound at the L3L4 site, possibly complexed with LPS. Under normal conditions, phospholipids are confined to the inner leaflet of the outer membrane, and cannot reach the active site of the enzyme. Under the influence of a membrane perturbation, substrate becomes available in the outer leaflet. This presumably triggers dimerisation of OMPLA in vivo, leading to the formation of a dimer with a high-affinity catalytic calcium-binding site. Subsequent transfer of calcium from the L3L4 site to this catalytic site results in the active enzyme dimer, which can productively bind and hydrolyse substrate. A second calcium ion may be bound in the now empty binding L3L4 site in each monomer, yielding a dimer complex with, in total, four calcium ions, as has been found in gel-filtration experiments. ${ }^{21}$ Thus far, structural evidence for this latter complex is missing due to practical limi- tations encountered with crystal soaking and manipulation.

The key elements of this model, i.e. calciumbinding and activation, are conserved; amino acid conservation extends beyond the catalytic residues and includes the catalytic calcium-binding site and the dimerisation face of the enzyme. This suggests that the architecture of all OMPLA enzymes is most likely the same, and that their activity is calcium and dimerisation-dependent. Several of the conserved residues are close in sequence, and form a complete and highly specific consensus sequence motif, $Y T Q-\mathrm{X}_{n}-\mathrm{G}-\mathrm{X}_{2}-\mathrm{H}-\mathrm{X}-\mathrm{SNG}$. This motif proves to be much more specific than a motif based on the active-site residues alone, ${ }^{7}$ which in addition to the OMPLAs finds 17 false positives in a profile search.

The residues that coordinate the L3L4 calcium ion are not preserved in the Neisseria and Helicobacter OMPLA sequences. However, this calcium ion does not seem to be essential for the catalytic mechanism, and it is conceivable that calcium can be sequestered from the environment in different ways. Furthermore, the outer membrane composition of Neisseria and H. pylori differs substantially from that of E. coli. In Neisseria, phospholipid molecules are present in the outer leaflet, ${ }^{34}$ while the outer membrane of $H$. pylori can contain substantial amounts of lyso-phospholipids. ${ }^{35}$ The lack of an L3L4 site in these OMPLAs may be related to these differences in membrane composition. Alternatively, the Helicobacter and Neisseria OMPLAs, which are least homologous to E. coli, may still have an L3L4 calcium-binding site that is obscured by sequence differences and insertions.

\section{Conclusions}

This study describes the structures of monomeric OMPLA complexed with calcium and the calciumfree inhibited dimeric OMPLA. The results demonstrate that the calcium-binding site in monomeric OMPLA (L3L4 site) is different from the catalytically important calcium in dimeric OMPLA. The monomeric form of OMPLA has one calciumbinding site and, only after dimerisation, a second calcium-binding site (one per monomer) is formed. The L3L4 calcium-binding site is preformed and calcium-binding at this site is not accompanied by structural rearrangements. Similarly, calciumbinding in dimeric OMPLA does not induce conformational changes. Thus, in dimeric OMPLA, the catalytic calcium-binding site is also preformed. Whereas the role of the catalytic calcium ion is evident, the function of the L3L4 calcium ion is not known. We postulate that this calcium ion could serve as a sink for calcium ions, and that it might complex with the LPS matrix, hence maintaining the barrier function of the outer membrane. Further study is required to test these hypotheses. 


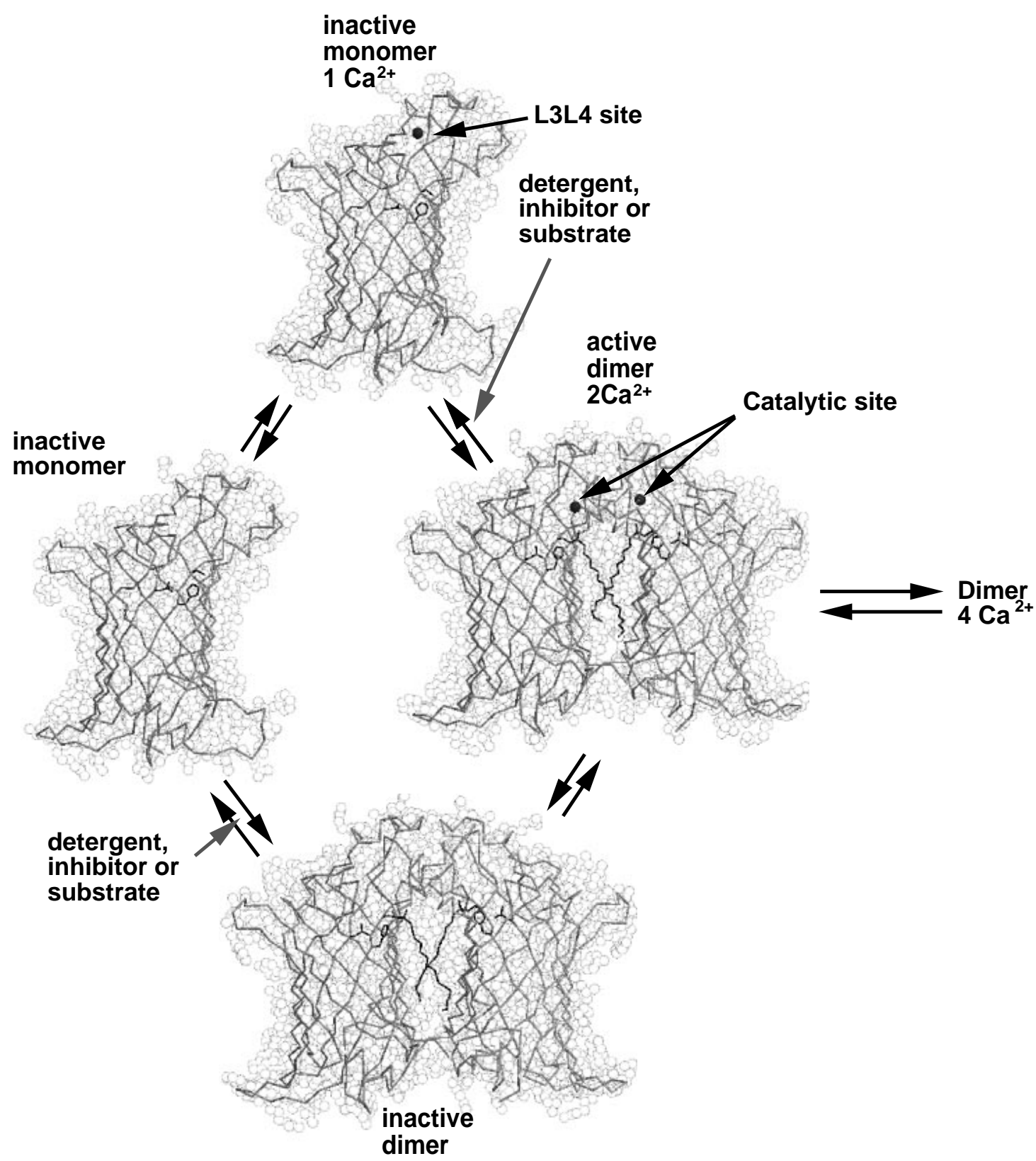

Figure 6. Model for complex formation of OMPLA. The $\mathrm{C}^{\alpha}$-trace and a CPK representation of the OMPLA structures are depicted. The calcium ions are shown as black spheres and the active-site residues and inhibitor are depicted as stick models. The differenct calcium-binding sites are labelled L3L4-site and the catalytic site for monomeric and dimeric OMPLA, respectively. Dimerisation is influenced by the environmental conditions, i.e. detergent, inhibitor hexadecanesulfonyl binding and substrate. Possible pathways are indicated that lead to the active species, either dimer with the catalytic calcium bound or dimer with calcium ions bound in both the catalytic site and the L3L4 site.

\section{Materials and Methods}

\section{Structure determination}

Outer membrane phospholipase A (OMPLA) was purified and refolded to its native state as described. ${ }^{36}$ Crystallisation of the monomeric form of OMPLA was performed as reported by Blaauw et al.,37 except that macro-seeding was used. Crystals were transferred to a stabilising mother liquor containing $27-29 \%$ (v/v) 2-methyl-2,4-pentanediol (MPD), $1.5 \% \quad(\mathrm{w} / \mathrm{v})$ 1-O-n-octyl- $\beta$-D-glucopyranoside ( $\beta$-OG), $1 \mathrm{mM} \mathrm{CaCl}{ }_{2}$ and $0.1 \mathrm{M}$ Bis-Tris buffer at $\mathrm{pH}$ 5.9. As no bound calcium could be identified in the crystals in the presence of $1 \mathrm{mM} \mathrm{CaCl}_{2}$, crystals were soaked for 30 minutes in the stabilising mother liquor with $50 \mathrm{mM} \mathrm{CaCl}_{2}$. The calcium-soaked crystals belong to space group $P 3_{1} 21$ with cell dimensions $a=b=77.53 \AA$ and $c=100.53 \AA$. Crystals of OMPLA with bound $\mathrm{Sm}^{3+}$ were obtained by cocrystallisation using as precipitant $25-28 \%(\mathrm{v} / \mathrm{v}) \mathrm{MPD}$, 
$1.5 \%(\mathrm{w} / \mathrm{v}) \beta-\mathrm{OG}, 0.3-2.0 \mathrm{mM} \mathrm{SmCl}_{3}$ and $0.1 \mathrm{M}$ Bis-Tris buffer at $\mathrm{pH}$ 5.9. These crystals also belonged to space group $P 3_{1} 21$ and had cell dimensions $a=b=78.68 \AA$ and $c=101.93 \AA$. Crystals of the dimeric enzyme were obtained from protein material inactivated by hexadecanesulfonylfluoride. ${ }^{38}$ Crystals were grown at $20^{\circ} \mathrm{C}$ in hanging drops with a starting solution containing $10 \mathrm{mg} / \mathrm{ml}$ inhibited-OMPLA, $1.95 \%$ (w/v) $\beta-O G$, $10 \mathrm{mM} \mathrm{KCl}$ and $2 \mathrm{mM}$ Tris at $\mathrm{pH}$ 6.6, equilibrated against $12-17 \%$ (v/v) PEG400 or $0.25 \mathrm{M}$ ammonium phosphate. These crystals were flash-frozen using $70 \%$ $(\mathrm{w} / \mathrm{w})$ phytohistol (Roth, Germany) in $0.25 \mathrm{M}$ ammonium phosphate as a cryoprotectant. The crystals belong to space group $P 22_{1} 2_{1} 2_{1}$ with cell dimensions $a=81.54 \AA, b=84.97 \AA$ and $c=95.80 \AA$, and have two molecules in the asymmetric unit.

\section{Data collection and phasing}

All data were recorded at $120 \mathrm{~K}$ using the stabilising mother liquor or the phytohistol solution as cryoprotectant. Data for the calcium-bound monomeric and dimeric form of OMPLA were collected using a MAR345 detector, at the protein crystallography beamline at the ELETTRA synchrotron, Triëste, and at the BW7B beamline of the EMBL-outstation at DESY in Hamburg, respectively. The raw data were processed with the programs DENZO, SCALEPACK ${ }^{39}$ and TRUNCATE. ${ }^{40}$ Data for the samarium-bound monomeric form were collected using a FAST area-detector (Nonius, Delft) equipped with a CAD4 к-goniostat, with graphite monochromatised $\mathrm{CuK} \alpha$ radiation from a Nonius FR571 rotating anode generator as X-ray source. Data collection and reduction was done with the MADNES system, ${ }^{41}$ profile fitting of the data was according to Kabsch. ${ }^{42}$ Data were scaled and merged using the Groningen BIOMOL crystallographic software package. Phases from the refined model of calcium-free monomeric OMPLA (PDB entry code $1 Q D 5^{22}$ ) were used for the calculation of the difference Fourier map and the anomalous difference Fourier map with the samarium data. Phase information for the dimeric OMPLA was obtained in a trivial case of mol- ecular replacement using $\mathrm{AMoRe}^{40}$ with the monomer as search model.

\section{Refinement}

The monomer model was refined using the conjugate gradient positional refinement in $\mathrm{XPLOR}^{43}$ cycled with minor manual rebuilding using O. ${ }^{44}$ Bulk solvent correction, overall anisotropic $B$-factor scaling, and a resolution-dependent weighting scheme were applied, and temperature factors were refined individually. Fifty water molecules and one calcium ion were included in the model. Remaining difference density around the protein molecule was interpreted as disordered detergent. Of the main-chain phi-psi torsion angles, $82.5 \%$ are in the most favoured regions, while none is in the disallowed regions of the Ramachandran plot. The final $R$ factor and free $R$-factor are $22.2 \%$ and $29.8 \%$, respectively (Table 1 ). The dimer model was refined using the conjugate gradient positional refinement in XPLOR as well. ${ }^{43}$ Bulk solvent correction, overall anisotropic $B$-factor scaling, and a resolution-dependent weighting were applied. Initially, tight non-crystallographic symmetry (NCS) restraints were applied to the two molecules in the asymmetric unit, but gradually these NCS restraints were released. No NCS restraint was associated with residues $44,71,103,104,125,126,128,138,140,142,144$, 156 , or 220 , where clearly deviating electron density was present. Restrained individual temperature factor refinement resulted in a final $R$ of $22.4 \%$ and $R_{\text {free }}$ of $28.1 \%$.

The final monomeric model contains residues 13-269, the dimeric model contains residues 13-23 and 31-269 of both monomers in the asymmetric unit. The overall geometry of both models is good (see Table 1) and none of the residues has disallowed main-chain phi, psi torsion angles in the Ramachandran plots. The r.m.s. coordinate errors are $0.3 \AA$ as estimated from a sigmaA plot for both the monomeric and dimeric structures. Temperature factors are relatively high, with values up to $100 \AA^{2}$ for side-chains in loops. This corresponds with high overall $B$-factors as determined from a Wilson plot.

Table 1. Data collection and refinement statistics

\begin{tabular}{|c|c|c|c|}
\hline & \multicolumn{2}{|c|}{ Monomeric OMPLA } & \multirow[t]{2}{*}{ Dimeric OMPLA } \\
\hline & $\mathrm{Ca}^{2+}$ & $\mathrm{Sm}^{3+}$ & \\
\hline Space group & $P 3_{1} 21$ & $P 3_{1} 21$ & $P 2{ }_{1} 2_{1} 2_{1}$ \\
\hline Resolution $(\AA)$ & $30-2.6(2.64-2.60 \AA)$ & $34-3.3$ (3.4-3.3 А̊) & $37.9-2.8(2.90-2.79 \AA)$ \\
\hline Unit cell $(a, b, c)(\AA)$ & $77.53,77.53,100.53$ & $78.68,78.68,101.93$ & $81.54,84.97,95.80$ \\
\hline Reflections & 47,201 & 20,753 & 53,984 \\
\hline Unique reflections & 9975 & 5793 & 14,611 \\
\hline Completeness (\%) & $89.2(62.3)$ & $97.3(90.2)$ & $81.0(53.5)$ \\
\hline Completeness of Friedel pairs (\%) & & $91.0(74.3)$ & \\
\hline$\langle I\rangle /\langle\sigma\rangle$ & $37.3(10.3)$ & $14.0(5.8)$ & $8.3(2.9)$ \\
\hline$R_{\mathrm{sym}}(\%)$ & $3.4(8.4)$ & $5.3(10.9)$ & $12.1(28.6)$ \\
\hline Refinement & \multicolumn{2}{|c|}{ Monomeric OMPLA Ca ${ }^{2+}$} & Dimeric OMPLA \\
\hline Protein atoms & \multicolumn{2}{|c|}{2094} & 3119 \\
\hline$R$-factor $(\%)$ & \multicolumn{2}{|c|}{$22.2(33.6)$} & $22.4(24.7)$ \\
\hline$R_{\text {free }}(\%)$ & \multicolumn{2}{|c|}{$29.8(38.5)$} & $28.1(33.2)$ \\
\hline$B$-factor $\left(\AA^{2}\right)$ & \multicolumn{2}{|c|}{49.9} & 37.9 \\
\hline Non-protein atoms & \multicolumn{2}{|c|}{51} & 0 \\
\hline Error estimate $(\AA)$ & \multicolumn{2}{|c|}{0.4} & 0.3 \\
\hline r.m.s.d. bond lengths ( $(̊)$ & \multicolumn{2}{|c|}{0.008} & 0.010 \\
\hline r.m.s.d. bond angle (deg.) & \multicolumn{2}{|c|}{1.2} & 1.4 \\
\hline r.m.s.d. dihedrals (deg.) & \multicolumn{2}{|c|}{26.4} & 27.3 \\
\hline r.m.s.d. improper dihedrals (deg.) & \multicolumn{2}{|c|}{1.23} & 1.19 \\
\hline
\end{tabular}

The numbers in parentheses are for the outermost shell of data. 
Screening the databases of genomic sequencing projects was performed with BLAST. ${ }^{45}$ Preliminary sequence data for Salmonella typhi, Yersinia pestis, Bordetella pertussis was obtained from the Sanger Centre website (http://www.sanger.ac.uk), funded by Beowulf Genomics. Sequence data for Pasteurella multocida was obtained from the University of Minnesota genome sequencing website, (http://www.cbc.umn.edu/ ResearchProjects/AGAC/Pm/Index.html), supported by the USDA-NRI, Minnesota Agricultural Experiment Station and the Minnesota Turkey Research and Promotion Council. Sequence data for Neisseria gonorrhoeae was obtained from OU-ACGT, Gonococcal Genome Sequencing Project supported by USPHS/NIH grant number AI38395. Sequence data of Salmonella enteriditis were produced by The Department of Microbiology at the University of Illinois and were obtained from the website http://www.salmonella.org. The OMPLA sequences were aligned with CLUSTALX ${ }^{46}$ and are presented in Figure 5 using the program ALSCRIPT. ${ }^{47}$ Figures 1, 3, 4 and 6 were produced using MOLSCRIPT ${ }^{48}$ and BOBSCRIPT. ${ }^{49}$

\section{Protein Data Bank accession codes}

The coordinates of the model structures have been deposited with the RCSB Protein Data Bank with accession codes 1FW2 and 1FW3.

\section{Acknowledgements}

We gratefully thank the staff of the EMBL outstation at DESY, Hamburg, Germany, and the staff of the protein crystallography beamline at the ELETTRA synchrotron, Triëste, for assistance during data collection. We thank all the universities and institutions who made sequence data available prior to publication and we thank all those organisations that funded these genome sequencing projects. We thank the European Union for support of the work through the HCMP Access to Large Installations Project, contract number CHGE-CT 93-0040. The investigations were supported by the Netherlands Foundation for Chemical Research $(\mathrm{CW})$ with financial aid from the Netherlands Organisation for Scientific Research (NWO).

\section{References}

1. Scandella, C. J. \& Kornberg, A. (1971). A membranebound phospholipase A1 purified from Escherichia coli. Biochemistry, 10, 4447-4456.

2. Homma, H., Chiba, N., Kobayashi, T., Kudo, I., Inoue, K., Ikeda, H., Sekiguchi, M. \& Nojima, S. (1984). Characteristics of detergent-resistant phospholipase A overproduced in E. coli cells bearing its cloned structural gene. J. Biochem. 96, 1645-1653.

3. Homma, H., Kobayashi, T., Chiba, N., Karasawa, K., Ikeda, H., Sekiguchi, M. \& Nojima, S. (1984). The DNA encoding pldA gene, the structural gene for detergent-resistant phospholipase A of E. coli. J. Biochem. 96, 1655-1664.

4. Nishijima, M., Nakaike, S., Tamori, Y. \& Nojima, S. (1977). Detergent-resistant phospholipase A of Escherichia coli K-12. Eur. J. Biochem. 73, 115-124.

5. Horrevoets, A. J. G., Hackeng, T. M., Verheij, H. M., Dijkman, R. \& De Haas, G. H. (1989). Kinetic charac- terization of Escherichia coli outer membrane phospholipase A using mixed detergent-lipid micelles. Biochemistry, 28, 1139-1147.

6. Brok, R. G. P. M., Brinkman, E., van Boxtel, R., Bekkers, A. C., Verheij, H. M. \& Tommassen, J. (1994). Molecular characterization of enterobacterial pldA genes encoding outer membrane phospholipase A. J. Bacteriol. 176, 861-870.

7. Brok, R. G. P. M., Boots, A. P., Dekker, N., Verheij, H. M. \& Tommassen, J. (1998). Sequence comparison of outer membrane phospholipases A: implications for structure and for the catalytic mechanism. Res. Microbiol. 149, 703-710.

8. Grant, K. A., Ubarretxena-Belandia, I., Dekker, N., Richardson, P. T. \& Park, S. F. (1997). Molecular characterization of $p l d A$, the structural gene for a phospholipase A from Campylobacter coli, and its contribution to cell-associated hemolysis. Infect. Immun. 65, 1172-1180.

9. Dekker, N. (2000). Outer-membrane phospholipase A: known structure, unknown biological function. Mol. Microbiol. 35, 711-717.

10. Pugsley, A. P. \& Schwartz, M. (1984). Colicin E2 release: lysis, leakage or secretion? Possible role of a phospholipase. EMBO J. 3, 2393-2397.

11. Van der Wal, F. J., Luirink, J. \& Oudega, B. (1995). Bacteriocin release proteins: mode of action, structure, and biotechnological application. FEMS Microbiol. Rev. 17, 381-399.

12. Cavard, D., Baty, D., Howard, S. P., Verheij, H. M. \& Lazdunski, C. (1987). Lipoprotein nature of the colicin A lysis protein: effect of amino acid substitutions at the site of modification and processing. J. Bacteriol. 169, 2187-2194.

13. Lazdunski, C. J., Baty, D., Geli, V., Cavard, D., Morlon, J., Lloubes, R., Howard, S. P., Knibiehler, M., Chartier, M., Varenne, S., Frenette, M., Dasseux, J. L. \& Pattus, F. (1988). The membrane channelforming colicin A: synthesis, secretion, structure, action and immunity. Biochim. Biophys. Acta, 947, 445-464.

14. Dorrell, N., Celeste Martino, M., Stabler, R. A., Ward, S. J., Zhang, Z. W., McColm, A. A., Farthing, M. J. G. \& Wren, B. W. (1999). Characterization of Helicobacter pylori PldA, a phospholipase with a role in colonization of the gastric mucosa. Gastroenterology, 117, 1098-1104.

15. Audet, A., Nantel, G. \& Proulx, P. (1974). Phospholipase A activity in growing Escherichia coli cells. Biochim. Biophys. Acta, 348, 334-343.

16. Weiss, J., Beckerdite-Quagliata, S. \& Elsbach, P. (1979). Determinants of the action of phospholipases A on the envelope phospholipids of Escherichia coli. J. Biol. Chem. 254, 11010-11014.

17. Cronan, J. E., Jr \& Wulff, D. L. (1969). A role for phospholipid hydrolysis in the lysis of Escherichia coli infected with bacteriophage T4. Virology, 38, 241-246.

18. Luirink, J., van der Sande, C., Tommassen, J., Veltkamp, E., De Graaf, F. K. \& Oudega, B. (1986). Effects of divalent cations and of phospholipase A activity on excretion of cloacin DF13 and lysis of host cells. J. Gen. Microbiol. 132, 825-834.

19. Dekker, N., Tommassen, J., Lustig, A., Rosenbusch, J. P. \& Verheij, H. M. (1997). Dimerization regulates the enzymatic activity of Escherichia coli outer membrane phospholipase A. J. Biol. Chem. 272, 3179-3184.

20. Dekker, N., Tommassen, J. \& Verheij, H. M. (1999). Bacteriocin release protein triggers dimerization of 
outer membrane phospholipase A in vivo. J. Bacteriol. 181, 3281-3283.

21. Ubarretxena-Belandia, I., Boots, J. W. P., Verheij, H. M. \& Dekker, N. (1998). Role of the cofactor calcium in the activation of outer membrane phospholipase A. Biochemistry, 37, 16011-16018.

22. Snijder, H. J., Ubarretxena-Belandia, I., Blaauw, M., Kalk, K. H., Verheij, H. M., Egmond, M. R., Dekker, N. \& Dijkstra, B. W. (1999). Structural evidence for dimerization-regulated activation of an integral membrane phospholipase. Nature, 401, 717-721.

23. Read, R. J. (1986). Improved Fourier coefficients for maps using phases from partial structures with errors. Acta Crystallog. sect. A, 42, 140-149.

24. Cygler, M., Grochulski, P., Kazlauskas, R. J., Schrag, J. D., Bouthillier, F., Rubin, B., Serreqi, A. N. \& Gupta, A. K. (1994). A structural basis for the chiral preferences of lipases. J. Am. Chem. Soc. 116, 31803186.

25. Lang, D. A., Mannesse, M. L., de Haas, G. H., Verheij, H. M. \& Dijkstra, B. W. (1998). Structural basis of the chiral selectivity of Pseudomonas cepacia lipase. Eur. J. Biochem. 254, 333-340.

26. Nardini, M., Lang, D. A., Liebeton, K., Jaeger, K. E. \& Dijkstra, B. W. (2000). Crystal structure of Pseudomonas aeruginosa lipase in the open conformation. J. Biol. Chem. 275, 31219-31225.

27. Pautsch, A. \& Schulz, G. E. (1998). Structure of the outer membrane protein A transmembrane domain. Nature Struct. Biol. 5, 1013-1017.

28. Cowan, S. W., Schirmer, T., Rummel, G., Steiert, M., Ghosh, R., Pauptit, R. A., Jansonius, J. N. \& Rosenbusch, J. P. (1992). Crystal structures explain functional properties of two E. coli porins. Nature, 358, 727-733.

29. Kingma, R. L., Fragiathaki, M., Snijder, H. J., Dijkstra, B. W., Verheij, H. M., Dekker, N. \& Egmond, M. R. (2000). Unusual catalytic triad of Escherichia coli outer membrane phospholipase A. Biochemistry, 39, 10017-10022.

30. Kleywegt, G. J. \& Jones, T. A. J. (1996). Phi/Psi-chology: Ramachandran revisited. Structure, 4, 13951400.

31. Merck, K. B., de Cock, H., Verheij, H. M. \& Tommassen, J. (1997). Topology of the outer membrane phospholipase A of Salmonella typhimurium. J. Bacteriol. 179, 3443-3450.

32. Ferguson, A. D., Welte, W., Hofmann, E., Lindner, B., Holst, O., Coulton, J. W. \& Diederichs, K. (2000). A conserved structural motif for lipopolysaccharide recognition by procaryotic and eucaryotic proteins. Structure, 8, 585-592.

33. Norris, V., Grant, S., Freestone, P., Canvin, J., Sheikh, F. N., Toth, I., Trinei, M., Modha, K. \& Norman, R. I. (1996). Calcium signalling in bacteria. J. Bacteriol. 178, 3677-3682.

34. Lugtenberg, B. (1981). Composition and function of the outer membrane of Escherichia coli. Trends Biochem. Sci. 2, 262-265.

35. Bukholm, G., Tannœs, T., Nedenskov, P., Esbensen, Y., Grav, H. J., Hovig, T., Ariansen, S. \& Guldvog, I. (1997). Colony variation of Helicobacter pylori: pathogenic potential is correlated to cell wall lipid composition. Scan. J. Gastroenterol. 32, 445-454.

36. Dekker, N., Merck, K., Tommassen, J. \& Verheij, H. M. (1995). In vitro folding of Escherichia coli outermembrane phospholipase A. Eur. J. Biochem. 232, 214-219.
37. Blaauw, M., Dekker, N., Verheij, H. M., Kalk, K. H. \& Dijkstra, B. W. (1995). Crystallization and preliminary X-ray analysis of outer membrane phospholipase A from Escherichia coli. FEBS Letters, $373,10-12$.

38. Horrevoets, A. J., Verheij, H. M. \& de Haas, G. H. (1991). Inactivation of Escherichia coli outermembrane phospholipase A by the affinity label hexadecanesulfonyl fluoride. Evidence for an activesite serine. Eur. J. Biochem. 198, 247-253.

39. Otwinowski, Z. \& Minor, W. (1997). Processing of X-ray diffraction data collected in oscillation mode. Methods Enzymol. 276, 307-326.

40. Collaborative Computational Project Number 4 (1994). The CCP4 suite: programs for protein crystallography. Acta Crystallog. sect. D, 50, 760-763.

41. Messerschmidt, A. \& Pflugrath, J. W. (1987). Crystal orientation and X-ray pattern prediction routines for area-detector diffraction systems in macromolecular crystallography. J. Appl. Crystallog. 20, 306-315.

42. Kabsch, W. (1988). Evaluation of single crystal X-ray diffraction data from a position sensitive detector. J. Appl. Crystallog. 21, 916-924.

43. Brünger, A. T. (1992). Free $R$ value: a novel statistical quantity for assessing the accuracy of structures. Nature, 355, 472-475.

44. Jones, T. A., Zou, J.-Y., Cowan, S. W. \& Kjeldgaard, M. (1991). Improved methods for building protein models in electron density maps and the location of errors in these models. Acta Crystallog. sect. A, 47, 110-119.

45. Altschul, S. F., Madden, T. L., Schiffer, A. A., Zhang, J., Zhang, Z., Miller, W. \& Lipman, D. J. (1997). Gapped BLAST and PSI-BLAST: a new generation of protein data base search programs. Nucl. Acids Res. 25, 3389-3402.

46. Thompson, J. D., Higgins, D. G. \& Gibson, T. J. (1994). CLUSTAL W: improving the sensitivity of progressive multiple sequence alignment through sequence weighting, position-specific gap penalties and weight matrix choice. Nucl. Acids Res. 22, 46734680.

47. Barton, G. J. (1993). ALSCRIPT: a tool to format multiple sequence alignments. Protein Eng. 6, 37-40.

48. Kraulis, P. J. (1991). MOLSCRIPT: a program to produce both detailed and schematic plots of protein structures. J. Appl. Crystallog. 24, 946-950.

49. Esnouf, R. M. (1997). An extensively modified version of Molscript that includes greatly enhanced coloring capabilities. J. Mol. Graph. 15, 132-134.

50. Parkhill, J., Wren, B. W., Mungall, K., Ketley, J. M., Churcher, C., Basham, D., Chillingworth, T., Davies, R. M., Feltwell, T., Holroyd, S., Jagels, K., Karlyshev, A. V., Moule, S., Pallen, M. J., Penn, C. W. et al. (2000). The genome sequence of the food-borne pathogen Campylobacter jejuni reveals hypervariable sequences. Nature, 403, 665-668.

51. Tomb, J. F., White, O., Kerlavage, A. R., Clayton, R. A., Sutton, G. G., Fleischmann, R. D., Ketchum, K. A., Klenk, H. P., Gill, S., Dougherty, B. A., Nelson, K., Quackenbush, J., Zhou, L. X., Kirkness, E. F., Peterson, S. et al. (1997). The complete genome sequence of the gastric pathogen Helicobacter pylori. Nature, 389, 412-422.

52. Alm, R. A., Ling, L. S. L., Moir, D. T., King, B. L., Brown, E. D., Doig, P. C., Smith, D. R., Noonan, B., Guild, B. C., deJonge, B. L., Carmel, G., Tummino, P. J., Caruso, A., Uria, Nickelsen M., Mills, D. M. et al. (1999). Genomic-sequence comparison of two 
unrelated isolates of the human gastric pathogen Helicobacter pylori. Nature, 397, 176-180.

53. Parkhill, J., Achtman, M., James, K. D., Bentley, S. D., Churcher, C., Klee, S. R., Morelli, G., Basham, D.,
Brown, D., Chillingworth, T., Davies, R. M., Davis, P., Devlin, K., Feltwell, T., Hamlin, N. et al. (2000). Complete DNA sequence of a serogroup A strain of Neisseria meningitidis Z2491. Nature, 404, 502-506.

Edited by I. B. Holland

(Received 12 January 2001; received in revised form 30 March 2001; accepted 3 April 2001) 